\title{
Papillary muscle relocation and annular repositioning for functional tricuspid regurgitation
}

\author{
Masashi Takeshita, MD, Hirokuni Arai, MD, PhD, Eiki Nagaoka, MD, PhD, and \\ Tomohiro Mizuno, MD, PhD, Tokyo, Japan
}

\footnotetext{
From the Department of Cardiovascular Surgery, Graduate School of Medical and Dental Sciences, Tokyo Medical and Dental University, Tokyo, Japan.

Disclosures: The authors reported no conflicts of interest.

The Journal policy requires editors and reviewers to disclose conflicts of interest and to decline handling or reviewing manuscripts for which they may have a conflict of interest. The editors and reviewers of this article have no conflicts of interest.

Received for publication May 11, 2020; revisions received May 11, 2020; accepted for publication May 18, 2020; available ahead of print May 24, 2020.

Address for reprints: Hirokuni Arai, MD, PhD, Department of Cardiovascular Surgery, Graduate School of Medical and Dental Sciences, Tokyo Medical and Dental University, 1-5-45 Yushima, Bunkyo-ku, Tokyo, Japan 113-8519 (E-mail: hiro.cvsg@tmd.ac.jp).

JTCVS Techniques 2020;3:162-5

2666-2507

Copyright $@ 2020$ The Authors. Published by Elsevier Inc. on behalf of The American Association for Thoracic Surgery. This is an open access article under the CC BY-NC-ND license (http://creativecommons.org/licenses/bync-nd/4.0/).

https://doi.org/10.1016/j.xjtc.2020.05.014
}

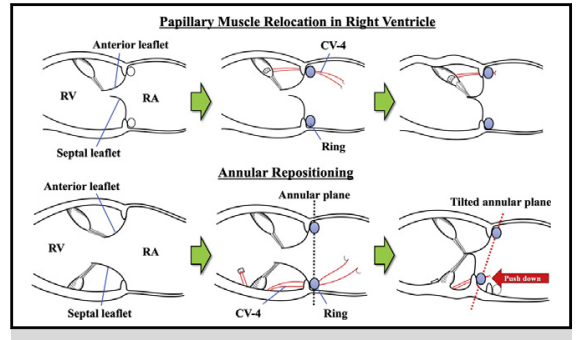

New subvalvular procedures for the treatment of TR with leaflet tethering.

CENTRAL MESSAGE

Subvalvular procedures of anterior papillary muscle relocation and septal annular repositioning were effective to relieve leaflet tethering and restore leaflet coaptation in severe functional TR.

See Commentaries on pages 166 and 168.
Tricuspid annuloplasty (TAP) is a standard procedure for treating functional tricuspid regurgitation (TR). However, for patients with severe leaflet tethering, the effectiveness of TAP is limited. ${ }^{1}$ We describe a case in which new subvalvular procedures were successfully applied in tricuspid valve repair to treat severe functional TR.

\section{CLINICAL SUMMARY AND SURGICAL TECHNIQUES}

This study was approved by our institution's ethical committee. A 66-year-old man with moderate ischemic mitral regurgitation, severe TR, coronary artery stenosis, and atrial fibrillation was referred to our hospital. He had significant lower-extremity edema and ascites. Preoperative transthoracic echocardiography (TTE) showed severe functional TR due to anterior and septal leaflet tethering, annular dilatation, and right ventricular (RV) dilatation, consequently leading to loss of leaflet coaptation (Figure 1, A). The left ventricular ejection fraction was $32 \%$. The RV fractional area change was $20 \%$. The tricuspid annular plane systolic excursion was $10.2 \mathrm{~mm}$. The tethering height was $15.0 \mathrm{~mm}$. Intraoperative findings of the tricuspid valve on beating heart revealed severe tethering of all leaflets and significant annular dilatation (Figure 1, B). We initially placed CV-4 polytetrafluoroethylene mattress sutures (Gore-Tex, Flagstaff, Ariz) in the anterior papillary muscle. The free ends of the CV-4 sutures were passed through the corresponding anterior annulus. After we performed TAP with a 32-mm Contour 3D ring (Medtronic, Minneapolis, Minn), the CV-4 sutures were also passed through the annuloplasty ring, and then pulled to relocate the anterior papillary muscle closer to the anterior annulus. The CV-4 sutures were tied when the anterior leaflet lifted up to the level of the tricuspid annular plane. This procedure, papillary muscle relocation in $\mathrm{RV}$, was effective in relieving the anterior leaflet tethering.

Edge-to-edge plication sutures between the septal and posterior leaflets had been placed; however, the saline test results showed remaining septal leaflet tethering. We subsequently placed additional CV-4 sutures in the RV septum. The free ends of the CV-4 sutures were passed through the corresponding septal annulus and annuloplasty ring. After saline injection into the RV, the sutures were tightened to reposition the septal annulus in the direction of the RV cavity. This additional procedure relieved septal leaflet 


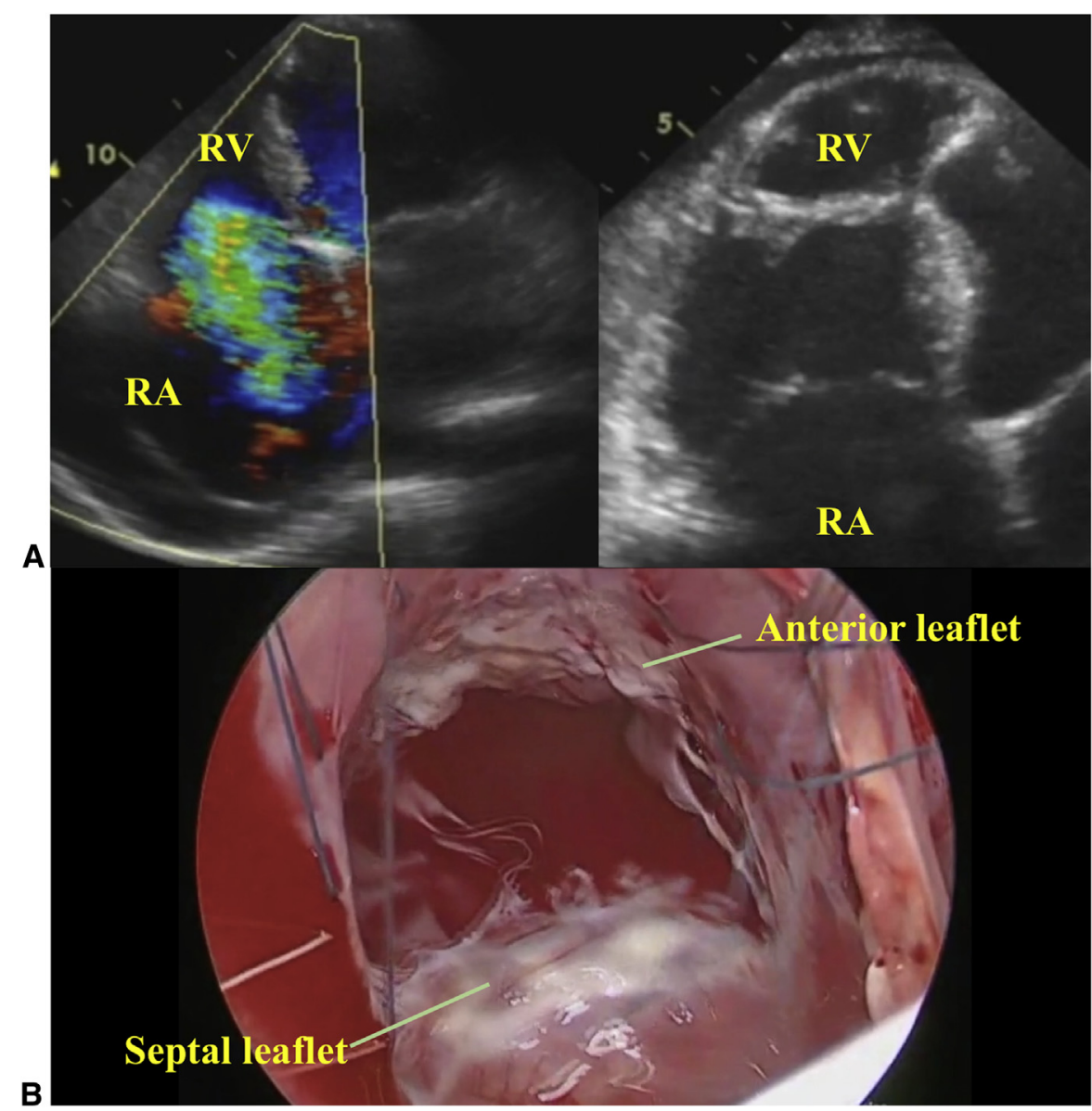

FIGURE 1. A, Preoperative echocardiogram showing regurgitation occurring due to annular dilatation and leaflet tethering (anterior and septal leaflet tethering [tethering height $15.0 \mathrm{~mm}$ ], annular dilatation [annular diameter $56.0 \mathrm{~mm}$ ], and right ventricular dilatation [right ventricular diastolic diameter $56.0 \mathrm{~mm}$, systolic diameter $49.2 \mathrm{~mm}]$ ). B, Intraoperative picture showing tethering of all leaflets. $R V$, Right ventricle; $R A$, right atrium.

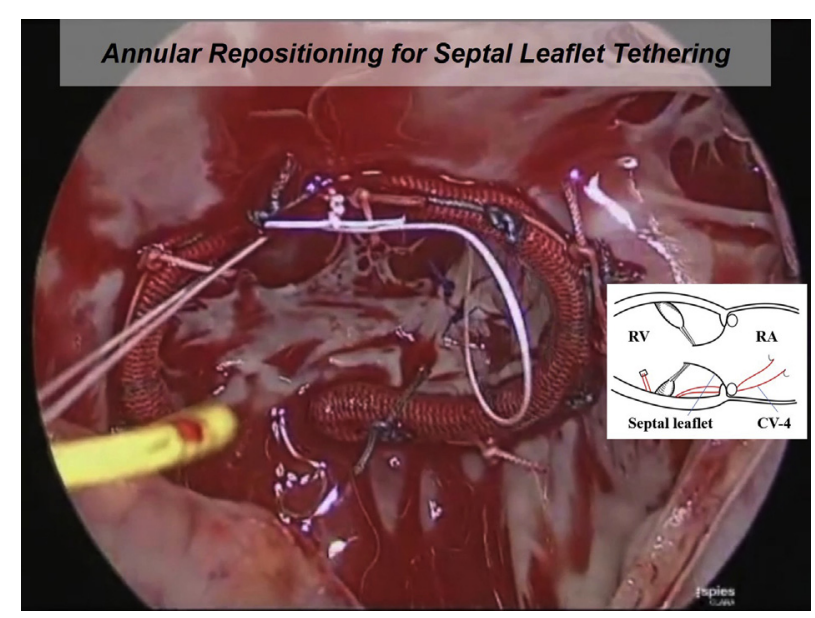

VIDEO 1. Video 1 demonstrates the papillary muscle relocation and annular repositioning procedures and transthoracic echocardiography performed preoperatively, postoperatively, and at 3 years postoperatively. Video available at: https://www.jtcvs.org/article/S2666-2507(20)30235-2/ fulltext. tethering, and the final saline test showed no significant regurgitation. We named this procedure "annular repositioning," which was effective for relieving septal leaflet tethering (Video 1). Mitral annuloplasty, coronary artery bypass grafting to the posterior descending branch using a saphenous vein graft, and the maze procedure were concomitantly performed. At 3 years postoperatively, TTE showed only mild TR with preserved leaflet motion without tricuspid stenosis.

\section{DISCUSSION}

We successfully applied new surgical techniques in tricuspid valve repair for the treatment of severe functional TR due to leaflet tethering. The concept of papillary muscle relocation is well known for the treatment of functional mitral regurgitation (Figure 2). We applied this concept to relieve the anterior leaflet tethering in severe functional TR and found that it was very effective. We apply this relocation technique when the anterior papillary muscle is a certain size and not separated into small multiheaded 


\section{Papillary Muscle Relocation in Right Ventricle}

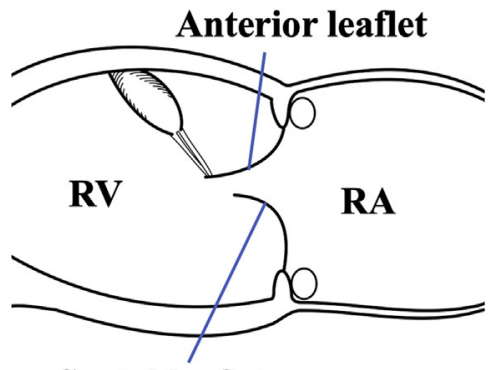

Septal leaflet
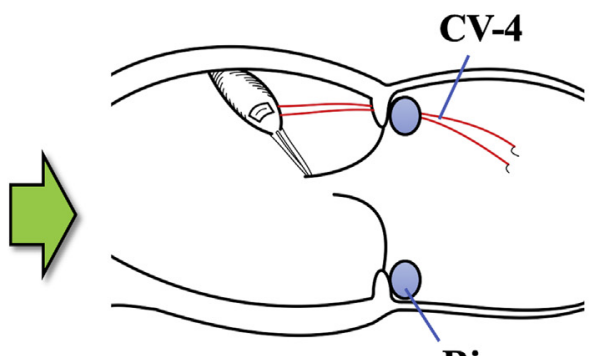

Ring

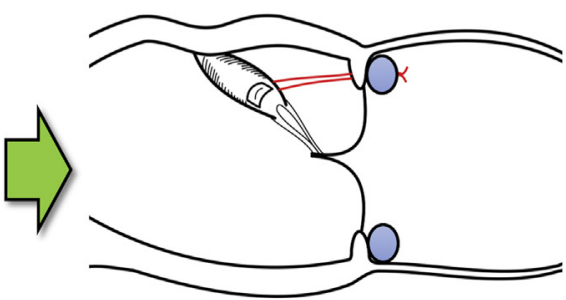

\section{Annular Repositioning}

\section{Anterior leaflet}

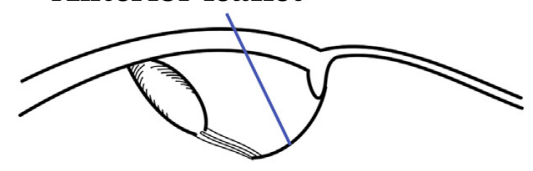

RV

RA

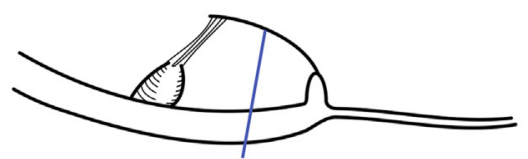

Septal leaflet
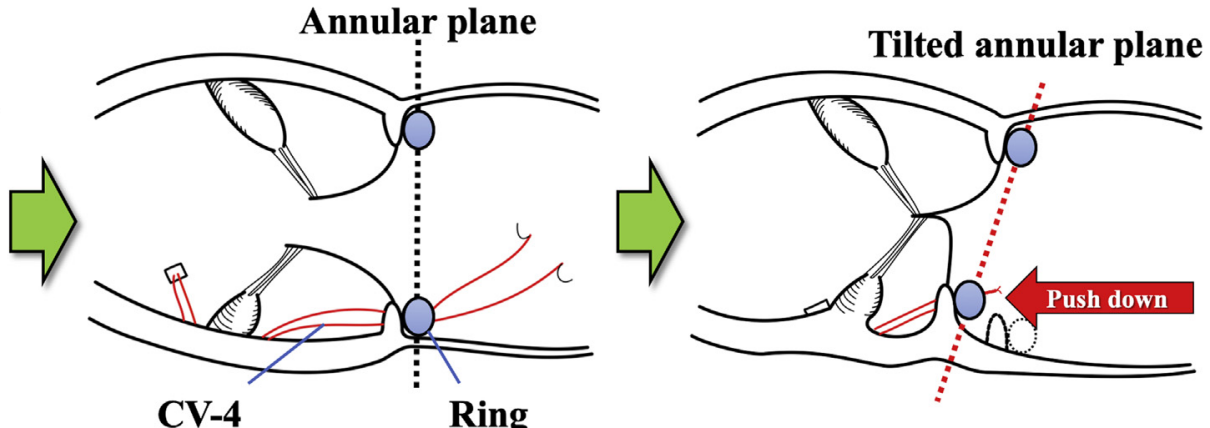

FIGURE 2. The concept of new subvalvular procedures for the treatment of TR with leaflet tethering. In anterior papillary muscle relocation, the CV-4 sutures are placed in the anterior papillary muscle. The free ends of them are passed through the corresponding anterior annulus. In annular repositioning, the CV-4 sutures are anchored to the RV septum around the origin of the papillary muscle or chords belonging to the septal leaflet. The free ends of them are passed through the corresponding septal annulus and ring. The degree of papillary muscle relocation and annular repositioning is determined based on the saline test results. During saline injection into the RV cavity with manual compression of the main pulmonary artery, the CV-4 sutures are tied when the anterior and septal leaflet approach the annular plane. By applying annular repositioning, the septal annulus relocates in the direction of the RV cavity. This maneuver leads to tilting of the annular plane with the rigid ring. As a result, the septal leaflet approaches the annular plane, and leaflet tethering is relieved. $R V$, Right ventricular; $R A$, right atrium.

muscles. Annular repositioning was another subvalvular procedure, the concept of which is new. In annular repositioning, the CV-4 sutures are anchored to the RV septum around the origin of the papillary muscle or chords belonging to the septal leaflet. The main purpose of this particular technique is to relieve the septal leaflet tethering by pushing the septal annulus into the RV cavity. After this initial case, we found it easier to put the CV-4 sutures before securing the annuloplasty ring.

Septal leaflet tethering is one of the most important factors of severe functional TR. Anatomically, the septal leaflet is the shortest and least mobile leaflet. ${ }^{2}$ Moreover, in a healthy individual, the lowest point of the tricuspid annulus is located at the septal segment near the posterior segment. In addition, the more severe the TR, the more planar the annulus becomes, and the septal segment is elevated in the direction of the right atrium. ${ }^{3}$ Thus, the short and the least mobile septal leaflet is susceptible to the relative elevation of the septal annulus and can be tethered easily. The concept of annular repositioning involves pushing the septal annulus into the RV cavity to return the septal annulus to its original position. The degree of leaflet tethering was measured using preoperative TTE. The tethering height, which is the distance between the coaptation of the septal and anterior leaflets and the tricuspid annular plane, was measured in the apical 4chamber view at mid-systolic phase. We defined the indication of these procedures as a tethering height of greater than $8 \mathrm{~mm}$ according to a previous report. ${ }^{4}$ In addition, the degree of papillary muscle relocation and annular repositioning was determined based on the saline test results. During saline injection into the RV cavity with manual compression of the main pulmonary artery, the CV-4 sutures were tied so that the anterior and septal leaflets floated above the RV cavity and the whole leaflets could be seen in the annular plane. In papillary muscle relocation for the anterior leaflet tethering, too much relocation should be avoided not to induce leaflet prolapse, because 
the anterior papillary muscle and corresponding RV free wall are easy to move. In this particular case, TR was controlled and leaflet motion was preserved at 3 years.

The size of the ring is routinely chosen according to the surface area of the anterior leaflet for TAP. By using these particular procedures in addition to anterior leaflet-sized ring annuloplasty, almost one half of the septal leaflet height is expected to result in the coaptation length between the anterior and septal leaflets. The height of the septal leaflet is reported to be 14.5 to $20.6 \mathrm{~mm}$ by Sakon and colleagues. ${ }^{5}$ Thus, the coaptation length between the anterior and septal leaflets would be more than $7 \mathrm{~mm}$, which is almost equal to one half of the septal leaflet height. The smaller-sized ring was not selected because Min and colleagues ${ }^{6}$ reported that leaflet tethering became aggravated after TAP and annulus size reduction could be achieved at the expense of aggravation of leaflet tethering.

Dreyfus and colleagues ${ }^{7}$ described anterior leaflet augmentation using an autologous pericardium. This is a useful technique to control TR with leaflet tethering. However, tricuspid stenosis due to late calcification of the autologous pericardium is a concern. The subvalvular procedures we introduced here are simple, do not require leaflet manipulations, and are not more demanding than leaflet-patching techniques.

Tricuspid valve replacement (TVR) might have been considered as an optional surgical procedure for this particular case. However, operative mortality of TVR is reported to be high. ${ }^{8}$ Because of the limited effects of TVR, tricuspid valve repair can be advocated if it is optimally performed. This is the initial case of anterior papillary muscle relocation and septal annular repositioning, and this patient was followed up for the longest period. We applied these subvalvular procedures for several cases after this particular case.
In conclusion, tricuspid valve repair with anterior papillary muscle relocation and septal annular repositioning in combination with rigid ring annuloplasty was a simple and effective procedure for functional TR due to severe leaflet tethering. These procedures can be applied in patients with the most severe type of functional TR, that is, in combination with tricuspid annular dilatation, leaflet tethering (tethering height of greater than $8 \mathrm{~mm}$ ), and loss of leaflet coaptation due to RV enlargement. Postoperatively, leaflet coaptation was restored with improved leaflet motion and satisfactory long-term durability. We believe that this technique will be one of the alternative surgical procedures for treating advanced functional TR.

\section{References}

1. Fukuda S, Song JM, Gillinov AM, McCarthy PM, Daimon M, Kongsaerepong V et al. Tricuspid valve tethering predicts residual tricuspid regurgitation after tricuspid annuloplasty. Circulation. 2005;111:975-9.

2. Dahou A, Levin D, Reisman M, Hahn RT. Anatomy and physiology of the tricuspid valve. JACC Cardiovasc Imaging. 2019;12:458-68.

3. Fukuda S, Saracino G, Matsumura Y, Daimon M, Tran H, Greenberg NL, et al Three-dimensional geometry of the tricuspid annulus in healthy subjects and in patients with functional tricuspid regurgitation: a real-time, 3-dimensional echocardiographic study. Circulation. 2006;114:I492-8.

4. Kim HK, Kim YJ, Park JS, Kim KH, Kim KB, Ahn H, et al. Determinants of the severity of functional tricuspid regurgitation. Am J Cardiol. 2006;15: 236-42.

5. Sakon Y, Murakami T, Fujii H, Takahashi T, Morisaki A, Yamane K, et al. New insight into tricuspid valve anatomy from 100 hearts to reappraise annuloplasty methodology. Gen Thorac Cardiovasc Surg. 2019;67:758-64.

6. Min SY, Song JM, Kim JH, Jang MK, Kim YJ, Song H, et al. Geometric changes after tricuspid annuloplasty and predictors of residual tricuspid regurgitation: a real-time three-dimensional echocardiography study. Eur Heart J. 2010;31: 2871-80.

7. Dreyfus GD, Raja SG, John Chan KM. Tricuspid leaflet augmentation to address severe tethering in functional tricuspid regurgitation. Eur J Cardiothorac Surg. 2008;34:908-10.

8. Kilic A, Saha-Chaudhuri P, Rankin JS, Conte JV. Trends and outcomes of tricuspid valve surgery in North America: an analysis of more than 50,000 patients from the Society of Thoracic Surgeons database. Ann Thorac Surg. 2013;96:1546-52. 